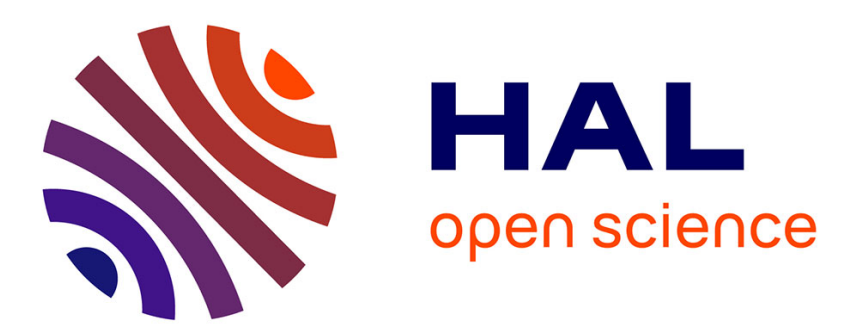

\title{
Relative and Absolute Positioning in Ultra Dense Mems System
}

Dermas Moffo, Philippe Canalda, François Spies

\section{To cite this version:}

Dermas Moffo, Philippe Canalda, François Spies. Relative and Absolute Positioning in Ultra Dense Mems System. International Conference on Computer and Information Technology, Dec 2016, Yanuca Island, Fiji. 10.1109/CIT.2016.95 . hal-02965132

\section{HAL Id: hal-02965132 \\ https://hal.science/hal-02965132}

Submitted on 13 Oct 2020

HAL is a multi-disciplinary open access archive for the deposit and dissemination of scientific research documents, whether they are published or not. The documents may come from teaching and research institutions in France or abroad, or from public or private research centers.
L'archive ouverte pluridisciplinaire HAL, est destinée au dépôt et à la diffusion de documents scientifiques de niveau recherche, publiés ou non, émanant des établissements d'enseignement et de recherche français ou étrangers, des laboratoires publics ou privés. 


\section{Relative and Absolute Positioning in Ultra Dense Mems System}

\author{
Dermas Moffo \\ Univ. Bourgogne Franche-Comté \\ FEMTO-ST Institute/CNRS \\ 25200 Montbéliard, FRANCE \\ Email: dermas.moffo@femto-st.fr
}

\author{
Philippe Canalda \\ Univ. Bourgogne Franche-Comté \\ FEMTO-ST Institute/CNRS \\ 25200 Montbéliard, FRANCE \\ Email: philipe.canalda@femto-st.fr
}

\author{
François Spies \\ Univ. Bourgogne Franche-Comté \\ FEMTO-ST Institute/CNRS \\ 25200 Montbéliard, FRANCE \\ Email: francois.spies@femto-st.fr
}

\begin{abstract}
Mems Microrobot applications evolve in ultradense contexts. They are at the stage of the simulation to obtain 2-Dimension or shapes for the deployment of individual or collective intelligent programs, and collectively achieve miniaturized and flexible workshops. Now-on, manufacturers and academicians projects are at the stage of modeling and prototyping Mems, and simulating Mems systems applications.

To tackle these objectives, addressing individual positioning of Mems inside very dense micro-systems becomes strategic. In many situations, it is more useful to know the relative positioning between Mems and their orientation, than the knowledge of absolute positioning of each Mems.

Works achieved on microrobots micropositioning are either stochastic or deterministic. The formers are based on probabilistic approaches, that provides better results on a small scale but produce greater error accumulation with a large sample. The latters are based on geometric considerations to accurately compute the position of each Mems, and then distribute it to all the elements.

We propose a model of a smart-grid (orthogonal and hexagonal lattice) of microrobots with regular geometry, and their connectors (actuators and sensors for moves and other actions) communicating by contact. Based on this model, we propose a mixed positioning algorithm (absolute and relative) in $2 \mathrm{D}$ without mobility of Mems in a group ranging in size from thousands to millions of items, based only on neighborly relations. Then by simulation we perform a functional validation of our algorithm, and a validation of the scalability of our algorithm on orthogonal grid of over 1 million node.
\end{abstract}

Keywords-2D Micro-localization; relative and global positioning; Micro-systems; localization algorithm; simulation;

\section{INTRODUCTION}

The miniaturization of the automated systems continues inexorably. With her, the densification of the systems reaches the million items in one $\mathrm{m}^{3}$, requiring new strategies of coordination. In such systems communications aspects between Mems, the individual and collective positionings of Mems, and finally the modelling of these systems constitute so many challenges in the academic and industrial communities.

In the sector of the communications for example, the development of new materials such as graphene, a leaf of the thickness of an atom, compound of atoms of carbon arranged according to a hexagonal motive, allows the manufacturing of new nano-devices. The frequency expected from nanotransmitters and nano-antennas made of graphene is of the order of the terahertz, giving rise to nano-wireless communications between microrobots[7] Microrobots endowed with these ways are still at the stage of the design. The current microrobots however communicate by contact and in real time when the classic robots communicate without contact but for very low densities (of the order of 2 in 6 robots by room). Micro-robots are used in various applications, as for the construction of intelligent 2D shape ( programmable matter - claytronics[4]), or still within the framework of miniaturized flexible workshops where elements collectively realize actions. All these applications require a precise knowledge of the position of the microrobot In many cases, it is more useful to have a local information about the relative positions of microrobots and their orientation, than a global position of every robot.

\section{A. Best poosition microrobots in systems with very high densities}

The two main issues of work on Mems systems relate, first carrying out a 2D shape with constraints resources or not (calculation time, completion time, energy consumption, percentage of achievement of shape goal), and then a succession of taken shape over time. Each taken shape can be completed with actions that can lead each Mems for individual or collective goal. In this context, the form of construction requires the knowledge of the positioning of Mems, or microrobots.

For the remainder of this work, we focus on the first issue, in other words the "static" and "instant" phase of the positioning of a micro-robots system, as opposed to a second, more dynamic phase that requires time synchronization of system actions. So many sub-issues appear. Among them, there is that of the knowledge of global and local (or relative) positioning of all Mems microrobots of a system, of all related components of a shape. There is also that of determining the position in an ultra-dense system of communicating Mems, which requires very reactive and efficient computational strategies. 


\section{B. Summary of our contributions of modeling algorithm and validation}

In the remainder of this paper, we propose at first a model of a microrobots smart-grid (orthogonal and hexagonal lattice) with regular or irregular geometry, and of their connectors (actuators and sensors for displacements and other actions) communicating through contact. Secondly, starting from this model, we propose a mixed positioning algorithm (absolute and relative) in 2D and 3D without mobility of Mems in a group ranging in size from thousands to million of items, based on neighborly relations depending on exchange links between microrobots. Thirdly, we perform a functional validation of our algorithm by simulations and a validation of the scalability of our algorithm on orthogonal grid of over 1 million nodes. Fourthly we analyze the results and present prospects for this work.

\section{THE POSITIONING A PRELIMINARY HYPOTHESIS}

Table I of the page 3 offers an analysis of the most representative works on Mems microrobots and Mems systems of the decade and who tackled the problem of individual massive positioning. This works are exclusively academic. They are structured from state projects or communities of states. The nature of work is still something of simulation and scaling faces on one hand the number of micro-robots that have to communicate and cooperate, and on the other hand, a quite relative functional and geometrical simplicity. As show by the data in the table, the maximum size of the robots grid does not exceed 10000 . In other works which cover the simulation of application of systems reconfiguration, the number may reach 400000 [1], but the calculation of positioning is collective (in groups of 3 catoms). It is handled in an implicit way applied in the context of a virtual territory discovery. The first target of this work [1] is the simulation of the Nano-wireless communications.

The data in the table also show that the size of Mems or microrobots elements considered range from a few $\mathrm{mm}^{3}$ to a few $d m^{3}$, that communication technologies are moving towards a wireless infrared communication (whereas previously the preferred communications technology between the robots were a matter of Bluetooth or WiFi). The data also reveal that the techniques of positioning are either probabilistic, either exact by neglecting the inherent uncertainties in the transmission of signal either still in the errors of mechanical or physical adjustments errors when switching to experiment with real prototypes. More important for our choices of orientations of works, positioning types are never mixted, that is to say, combining absolute positioning and the relative. Furthermore, the communication by contact is not understood in academia, the responsiveness of the positioning calculation is not measured in these dense systems of microrobots.

\section{Connectors, Mems, And Mems Systems}

In this section we present the modeling of a smart-grid (orthogonal and hexagonal lattice) of Mems with regular or irregular geometry, and their connectors (actuators and sensors for displacement and other actions) communicating through contat. We start by modeling the components and eventually those of the Mems system.

\section{A. In contact thanks to connectors}

A connector is a sensor/actuator enabling Mems to dock with each other. These connectors are also used for communication by contact, the energy transfer, solidification and dislocation of the material, and the movement of Mems.

For a Mems $M_{i}$, we model its $j-t h$ connector as follows:

$$
\text { Connector }_{i, j}=\left(C_{i, j}, \operatorname{PosR}\left(C_{i, j}, M_{i}\right), \text { Etat }_{i, j}, \text { Fonct }_{i, j}\right)
$$

In this formula, $C_{i, j}$ is the identifier of the connector $j$ of the Mems $M_{i}$ and $j$ given such as $\left.0 \leq j \leq \overline{(} C_{M_{i}}-1\right)$; where $C_{M_{i}}$ the set of connectors of Mems $M_{i}$ and $\bar{C}_{M_{i}}$ is the total number of connectors of the Mems $M_{i}$. $\operatorname{PosR}\left(C_{i, j}, M_{i}\right)$ is the relative position of the connector $C_{i, j}$ in the landmark $\mathcal{R}_{i}=\left(O_{M_{i}}, \overrightarrow{x_{M_{i}}}, \overrightarrow{y_{M_{i}}}\right)$ of the $M e m s_{i}$ and it's coordinates are given by:

$$
\operatorname{PosR}\left(C_{i, j}, M_{i}\right)=\left\{\begin{array}{l}
\left(d_{i, j}\left(O_{M_{i}}, C_{i, j}\right), \alpha_{i, j}\right) \text { polar } \\
\left(X_{C_{i, j}}, Y_{C_{i, j}}\left[, Z_{C_{i, j}}\right]\right) \text { cartesian }
\end{array}\right.
$$

Etat $_{i, j}$ the state of the connector $C_{i, j}$. A connector has a number of states that define its operation and the possible exchanges he has with the connectors of other Mems around him. We distinguish the following states: Let,s be $M_{i}$ a Mems and $\mathcal{R}_{i}=\left(O_{M_{i}}, \overrightarrow{x_{M_{i}}}, \overrightarrow{y_{M_{i}}}\right)$ the landmark associated to $M_{i}$; let's be a Mems $M_{k}$ and $\mathcal{R}_{k}=\left(O_{M_{k}}, \overrightarrow{x_{M_{k}}}, \overrightarrow{y_{M_{k}}}\right)$ the landmark associated to it.

- In contact: if it is in contact with another connector. Two connectors $C_{i, j} \in C_{M_{i}}$ and $C_{k, l} \in C_{M_{k}}$ are in contact if they are in physical contact. In this case, we can consider that they are at the same position.

$$
\left\{\begin{array}{l}
\operatorname{PosR}\left(C_{i, j}, M_{i}\right)=\operatorname{PosR}\left(C_{k, l}, M_{i}\right) \text { in } \mathcal{R}_{i} \\
\operatorname{PosR}\left(C_{i, j}, M_{k}\right)=\operatorname{PosR}\left(C_{k, l}, M_{k}\right) \text { in } \mathcal{R}_{k}
\end{array}\right.
$$

- contactless: if it is not in contact with another connector.

- connected: if connected to another connector. The connector $C_{i, j} \in C_{M_{i}}$ is connected to connector $C_{k, l} \in$ $C_{M_{k}}$ if $C_{i, j}$ can send and receive messages from $C_{k, l}$.

- disconnected: if there is no messages exchange with other neighboring connectors.

- active: if it is in a state allowing it to send or receive messages.

- inactive: if it can not send nor receive messages.

Fonct $_{i, j}$ the features of the connector $C_{i, j}$. A connector is able to perform several functions: sense, act, attract, repelling, send messages, receive messages, hang, lift, dock, undock. 


\begin{tabular}{|c|c|c|c|c|c|c|c|c|c|}
\hline \multirow{2}{*}{ Ref } & \multirow{2}{*}{$\begin{array}{l}\text { Positioning } \\
\text { strategy }\end{array}$} & \multirow{2}{*}{$\begin{array}{l}\text { Work } \\
\text { category }\end{array}$} & \multirow{2}{*}{ Work's type } & \multirow{2}{*}{$\begin{array}{l}\text { System } \\
\text { scalabil- } \\
\text { ity }\end{array}$} & \multirow{2}{*}{$\begin{array}{l}\text { Size of ele- } \\
\text { ment }\end{array}$} & \multicolumn{3}{|c|}{ Positioning } & \multirow{2}{*}{ communication } \\
\hline & & & & & & Method & Type & Reactivity & \\
\hline [5] & $\begin{array}{l}\text { use of the max- } \\
\text { imum likelihood } \\
\text { on a group of } \\
\text { robot }\end{array}$ & $\begin{array}{l}\text { Millibots } \\
\text { project }\end{array}$ & $\begin{array}{l}\text { simulation, } \\
\text { prototypage }\end{array}$ & $\begin{array}{l}\text { measures } \\
\text { made } 2 \text { - } \\
2 \text { between } \\
\text { several robots }\end{array}$ & - & - & - & - & $\begin{array}{l}\text { infrared, without } \\
\text { contact }\end{array}$ \\
\hline [9] & $\begin{array}{l}\text { positioning a } \\
\text { triangle of three } \\
\text { robots on a map }\end{array}$ & $\begin{array}{l}\text { scientific } \\
\text { project }\end{array}$ & simulation & 3 robots & undefined & $\begin{array}{l}\text { statistics } \\
\text { and } \\
\text { particles } \\
\text { filters }\end{array}$ & absolute & calculated & $\begin{array}{l}\text { infrared, without } \\
\text { contact }\end{array}$ \\
\hline$[8]$ & & $\begin{array}{l}\text { scientific } \\
\text { project }\end{array}$ & $\begin{array}{l}\text { simulation, } \\
\text { prototypage }\end{array}$ & i $2 \mathrm{~m}$ & $\begin{array}{l}28 * 28 * 30 \\
\mathrm{~cm}\end{array}$ & $\begin{array}{l}\text { exact cal- } \\
\text { culation }\end{array}$ & relative & calculated & $\begin{array}{l}\text { infrared, without } \\
\text { contact }\end{array}$ \\
\hline [3] & using module & $\begin{array}{l}\text { projet } \\
\text { Claytronics }\end{array}$ & $\begin{array}{l}\text { simulation, } \\
\text { prototypage }\end{array}$ & 10000 & millimeter & probabilistic & relative & calculated & $\begin{array}{l}\text { infrared, without } \\
\text { contactt }\end{array}$ \\
\hline [10] & & $\begin{array}{l}\text { Millibots } \\
\text { projet }\end{array}$ & $\begin{array}{l}\text { simulation, } \\
\text { prototypage }\end{array}$ & & $7 \times 7 \times 7 \mathrm{~cm}$ & $\begin{array}{l}\text { exact cal- } \\
\text { culation }\end{array}$ & & & $\begin{array}{l}\text { infrared, without } \\
\text { contact }\end{array}$ \\
\hline
\end{tabular}

Table I

PRINCIPAL PROJECTS AND ACADEMIC WORKS TACKLING POSITIONING WITHIN MICRO-ROBOT SYSTEMS

\section{B. Regular and irregular Mems with mixed positions}

A Mems is a micro-electromechanical system comprising one or more mechanical components using electricity as energy source in order to perform a function of sensor and/or actuator, with at least one structure having micrometric dimensions.

Physically, a Mems can have a shape more or less regular. We find Mems cubic, cylindrical, or spherical. In this work, we consider regular or irregular shapes Mems, with on their surface $\overline{C_{M_{i}}}$ connectors. We illustrate our work by $2 \mathrm{D}$ circular Mems having on their surface $\overline{C_{M_{i}}}$ regularly distributed connectors as shown in Figure Fig. 1.

Communication allows Mems to exchange messages, information, and ensure overall coordination of the Mems system. Several types of communication exist for Microrobots. According to the used medium, we find the wired, molecular, acoustic, electromagnetic, or contact communications.

Mems studied here, communicates by contact of neighbor with neighbor. They have connectors that are actuators also used for communication.

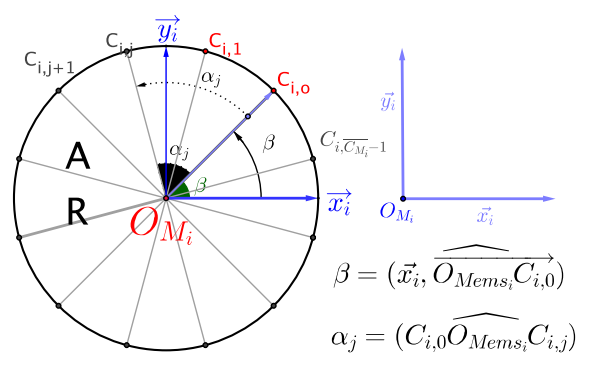
A : Absolute position
$0 \leq \mathrm{j} \leq \overline{\mathrm{C}_{\mathrm{M}_{\mathrm{i}}}}-1$
$\mathrm{R}$ : Relative position
- : No position (absolute ou relative)

Figure 1. Circular geometrical shape of homogeneous micro-robot with n-connectors - bi-dimensional projection
We give below the modelling of a Mems $\mathrm{Mems}_{i}$ :

$$
\begin{aligned}
\text { Mems }_{i}= & \left(M_{i}, O_{M_{i}}, \overrightarrow{x_{M_{i}}}, \overrightarrow{y_{M_{i}}}, \text { Geo }_{M_{i}}, C_{M_{i}}, \beta_{M_{i}},\right. \\
& \text { Fonct } \left._{M_{i}}, \text { Pos }_{M_{i}}, L_{M_{i}}, N_{M_{i}}\right)
\end{aligned}
$$

With :

* $i d_{M_{i}}:$ the identifier of $M e m s_{i}$.

$\star O_{M_{i}}$ : the center of the Mems $_{i}$

$\star \mathcal{R}_{i}=\left(O_{M_{i}}, \overrightarrow{x_{M_{i}}}, \overrightarrow{y_{M_{i}}}\right)$ : the local landmark associated with $M e m s_{i}$.

To define an orientation, we associate with every Mems $M e m s_{i}$ a local landmark $\mathcal{R}_{i}=\left(O_{M_{i}}, \overrightarrow{x_{i}}, \overrightarrow{y_{i}}\right)$.

We assume that the landmarks of all Mems have the same orientation.

Hypothesis: As represented on the figure Fig. 2, we formulate the following hypothesis:

$$
\begin{array}{r}
\forall \text { Mems }_{r}, \text { Mems }_{s}, r, s \in[0, \overrightarrow{\text { Mems }}-1], r \neq s, \\
\left\{\begin{array}{l}
\mathcal{R}_{r}=\left(O_{M_{r}}, \overrightarrow{x_{r}}, \overrightarrow{y_{r}}\right) \\
\mathcal{R}_{s}=\left(O_{M_{s}}, \overrightarrow{x_{s}}, \overrightarrow{y_{s}}\right)
\end{array}\right.
\end{array}
$$

, then

$$
\left\{\begin{array}{l}
\left(\overrightarrow{x_{r}}\right)_{\mathcal{R}_{r}}=\left(\overrightarrow{x_{s}}\right)_{\mathcal{R}_{r}}=\left(\overrightarrow{x_{s}}\right)_{\mathcal{R}_{s}}=\left(\overrightarrow{x_{r}}\right)_{\mathcal{R}_{s}} ; \\
\left(\overrightarrow{y_{r}}\right)_{\mathcal{R}_{r}}=\left(\overrightarrow{y_{s}}\right)_{\mathcal{R}_{r}}=\left(\overrightarrow{y_{s}}\right)_{\mathcal{R}_{s}}=\left(\overrightarrow{y_{r}}\right)_{\mathcal{R}_{s}}
\end{array}\right.
$$

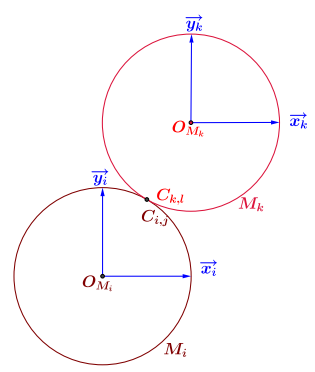

Figure 2. The vectors of local landmarks of neighboring robots are equal 
* $\mathrm{Geo}_{M_{i}}: \mathrm{Mems}_{i}$ geometry. Our modeling addresses Mems of any geometric shape. What is included in the geometry of the Mems is the position of its center, as well as the relative positions of the connectors relative to its center in the local landmark associated to the Mems.

* $C_{M_{i}}$ the set of connectors of $M e m s_{i}$. With $C_{M_{i}}=$ $\left\{C_{i, j},\right\}_{0 \leq j \leq \overline{C_{M_{i}}}-1}$; where $\overline{C_{M_{i}}}$ is the total number of connectors of every $M e m s_{i}$ and $C_{i, j}$ the $j-t h$ connector of $M e m s_{i}$. In the case of regular 2D circular Mems, the number $j$ of the connector allows to define its angular position

$\operatorname{PosR}\left(C_{i, j}, O_{M_{i}}\right)=\left(r_{M_{i}}, \alpha_{i, j}\right)$ with $\alpha_{i, j}=\frac{2 * j * \pi}{\overline{C_{M_{i}}}}$

$\star \beta_{M_{i}}$ Is the orientation angle of $M e m s_{i}$. It is defines by the angle of rotation of the connector of origin $C_{i, 0}$ in the landmark $\left(O_{M_{i}}, \overrightarrow{x_{M_{i}}}, \overrightarrow{y_{M_{i}}}\right)$ of $M e m s_{i}$

$\beta_{M_{i}}=\left(\overrightarrow{x_{M_{i}}, u_{i, 0}}\right)$ with $\overrightarrow{u_{i, 0}}=\overrightarrow{O_{M_{i}} C_{i, 0}}$

* Fonct $_{M_{i}}$ the features of the Mems $s_{i}$. A Mems has several features: communicate, move, run, load/unload, store.

* $\operatorname{Pos}_{M_{i}}$ the position of $M e m s_{i}$. Each Mems has an absolute position and a relative position. The absolute position is the position of its center in a common reference to all of our Mems system. We note it $\operatorname{PosA}_{M_{i}}$. The absolute position of a Mems Mems is given by

$$
\operatorname{Pos}_{M_{i}}=\left\{\begin{array}{l}
\left(d_{i}, \alpha_{i}\right) \text { polar coordinates } \\
\left(X_{M_{i}}, Y_{M_{i}}\right) \text { cartesian coordinates }
\end{array}\right.
$$

The position of Mems is a structure made up of an absolute position (when it exists) and of a list (empty or not) of relative positions.

* $L_{M_{i}}$ the set of $M e m s_{i}$ links. There is a link between two Mems if they have at least one pair of connector in contacts and connected. the Mems $M e m s_{i}$ il linked to the $\mathrm{Mems}_{k}$, if $\exists C_{i, j} \in C_{M_{i}}, \exists C_{k, l} \in C_{M_{k}}$ such as $C_{i, j}$ and $C_{k, l}$ are in contact and connected. All the links of the Mems $\mathrm{Mems}_{i}$ are note :

$$
\begin{aligned}
L_{M_{i}} & =\left\{\left(C_{i, j}, C_{k, l}, M_{k}\right) \text { with } C_{i, j} \in C_{M_{i}}, C_{k, l} \in C_{M_{k}},\right. \\
& \text { and } \left.C_{i, j} \text { in contact and connected to } C_{k, l}\right\}
\end{aligned}
$$

$\star N_{M_{i}}$ All the nearby Mems of Mems ${ }_{i}$.

$$
N_{M_{i}}=\left\{M_{k} / \exists\left(C_{i, j}, C_{k, l}, M_{r}\right) \in L_{M_{i}}, M_{r}=M_{k}\right\}
$$

\section{Mems system}

The Mems, because of their small size, cheap price and the fact that they are mass produced, millions of units can be used in very small spaces. The Distributed intelligents Mems systems consist of a large number of Mems, capable of fulfilling advanced tasks (shape construction), result of the addition of elementary actions of each of its constituent. Distributed Mems systems can be classified into two categories according to their topology: static or dynamic. Static distributed Mems have a fixed physical topology that will not change over time, while dynamic are composed of mobile units, making them dynamic logical topology. Mems systems can be categorized into different architectures based on the geometric arrangement of their nodes [12].

Lattice architectures have nodes which are arranged and connected in a regular manner, forming an orthogonal or hexagonal structure. This architecture allows an easy reconfiguration, bound to the nodes facility of movement in a finite set of positions of neighboring cells.

The chained architectures or in tree possess nodes that are connected in a chain topology or tree.

The mobile architectures possess nodes which lean on the environment to form more or less complex structures.

The relative arrangement of a node and its neighbors allows to define a regular grid structure. The prosition of a node and its neighbors to define a regular grid structure. The circular geometry of node forced to a maximum of 6 neighbors. When each node can have six neighbors, the set of possible positions of the nodes form a hexagonal lattice. In the situation where each node has at most 4 neighbors, an orthogonal lattice is obtained. This grid then defines all the possible positions that may occupy the nodes.

\section{Algorithm of ABSOlUte AND RELATIVE HYBRID POSITIONING}

Our proposed algorithm uses the communication by contact between Mems and cooperation between nearby Mems to spread information being used to the calculation of positions. It allows when in a related component, there is Mems having an absolute position, to calculate an absolute position for the set of Mems of this related component; and when there is not at least one Mems having an absolute position, to lean on Mems having a relative position, or just on the relation of neighborhood and the cooperation to define the relative positions of Mems.

Our positioning system works in the following way:

1) Initialization: choice of references Mems.

In a first stage, we choose Mems of reference. If the list of Mems with a known absolute position is not empty, we choose Mems in this list as Mems of reference. If this list is empty, we choose Mems of the lowest number as Mems of reference.

2) Construction of the neighborhood of reference Mems.

The following stage consists in building the neighborhood of Mems of reference. Each Mems possesses physical neighbors who are in physical contact with him; and connections which are communication channels between him and nearby Mems physically through their connectors. Thanks to its connectors and to their state, Mems knows if it has a connection or not. Thanks to the connections established with nearby Mems physically, Mems builds its table of 

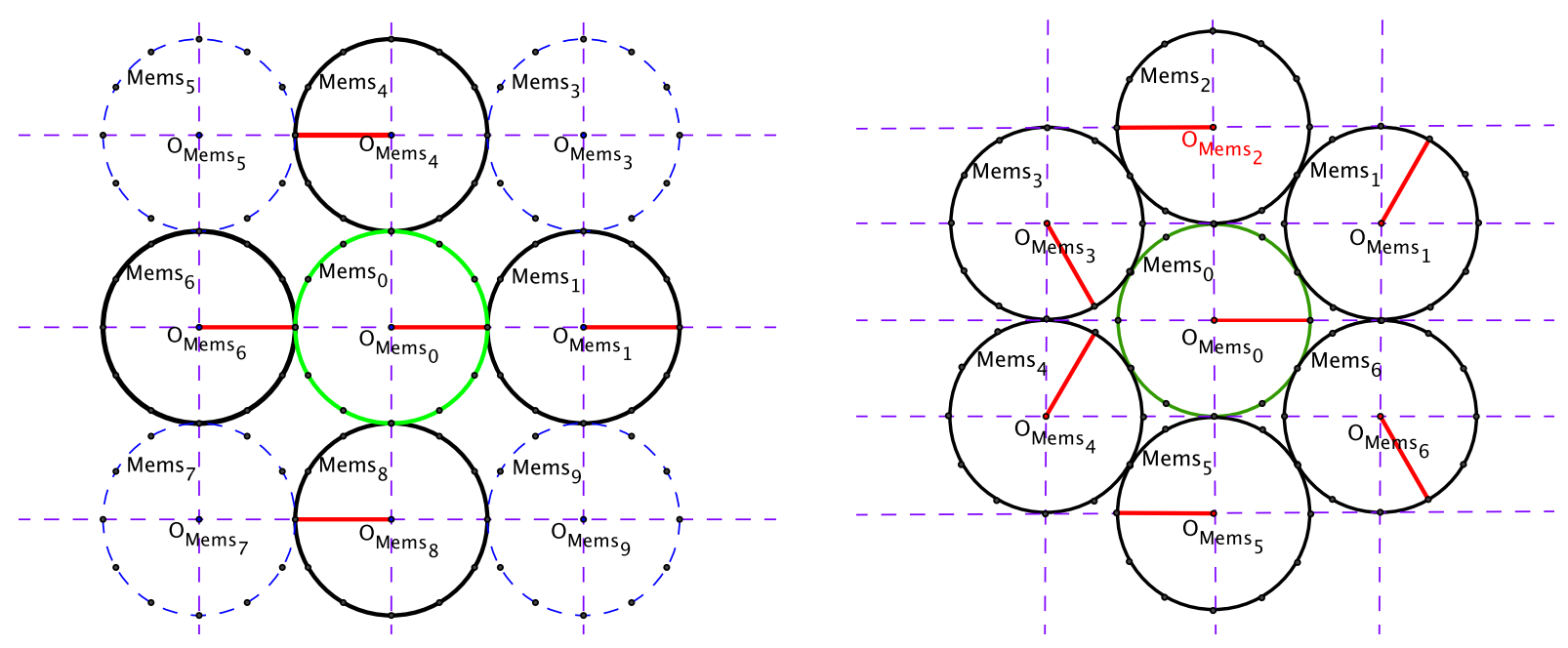

Figure 3. Configuration of orthogonal and hexagonal dense neighborhood of a Mems with circular geometry

neighborhood. The table of neighborhood of given Mems is so constituted by all the Mems which are neighbors to it physically, and in contact with at least one of its connectors through one of their connectors.

3) Calculation of the position of Mems of the neighborhood For every element of neighborhood we are going to calculate its relative position. The difficulty of this calculation lives on the fact that there is no global mark shared by all. Every mark of every Mems $\mathrm{Mems}_{i}$ being made of an origin situated in the center of Mems and made of vectors $\overrightarrow{x_{i}}, \overrightarrow{y_{i}}$. In this landmark, every connector has a relative position. We formulated hypotheses of simplification which allow us to calculate the relative position. Resting on the hypothesis of contact and considering that two connectors in contact occupy the same position. The calculation of the relative position is made as follows:

a) $\operatorname{Pos}_{M_{i}, M_{k}}=\left(\overrightarrow{O_{M_{i}} O_{M_{k}}}\right)_{\mathcal{R}_{i}}$

in the landmark $\mathcal{R}_{i}=\left(O_{M_{i}}, \overrightarrow{x_{i}}, \overrightarrow{y_{i}}\right)$ associated with $M e m s_{i}$

b) $\left(\overrightarrow{O_{M_{i}} O_{M_{k}}}\right)_{\mathcal{R}_{i}}=\left(\overrightarrow{O_{M_{i}} C_{i, j}}+\overrightarrow{C_{i, j} O M_{k}}\right)_{\mathcal{R}_{i}}$ using $C i, j$ applying the Chasles relationship

c) $\left(\overrightarrow{C_{i, j} O M_{k}}\right)_{\mathcal{R}_{i}}=\left(\overrightarrow{C_{k, l} O M_{k}}\right)_{\mathcal{R}_{i}}=$ $\left(\overrightarrow{C_{k, l} O M_{k}}\right)_{\mathcal{R}_{k}}$

$\mathcal{R}_{k}$, the landmark associated with Mems $M e m s_{k} ; C_{i, j}$ and $C_{k, l}$ being in contact we can consider them as being has the same position, thus we consider that the point $C_{i, j}$ is equal to the point $C_{k, l}$

d) $\left(\overrightarrow{C_{k, l} O M_{k}}\right)_{\mathcal{R}_{k}}=\left(-\overrightarrow{O M_{k} C_{k, l}}\right)_{\mathcal{R}_{k}}=-\operatorname{Pos}_{k, l}$

e) $\left(\overrightarrow{O_{M_{i}} C_{i, j}}\right)_{\mathcal{R}_{k}}=\operatorname{Pos}_{i, j}$

f) $\operatorname{Pos}_{i, k}=\operatorname{Pos}_{i, j}-\operatorname{Pos}_{k, l}$

4) Calculation return of the position of nearby Mems. This is made in the same ways the stage 3 . has been done.

5) Calculation of the Mems absolute position. The calculation of the absolute position leans on the broadcasting of information. Because of the weak resources which Mems has, our choice is the one not to use the usual techniques (dead recogning, ordometry) to calculate the absolute position. We consider that we possess within our system at least one Mems that the absolute position is known. Thanks to the previously calculated relative positions, Mems can determine its absolute position as follows: When it receives a message with the field absolute position not empty, having to determine the position relative as in (3), it uses it to calculate its absolute position as follows :

$$
\operatorname{Pos} A_{k}=\operatorname{Pos} A_{i}+\operatorname{PosR}(k, i)
$$

By correctly handling the removal of all the Mems that the absolute position have been calculated, we determine the absolute position of all Mems in the related component.

Thereafter our algorithm manipulates the following structures:

- List $<M E M S>L M$ : the list FIFO containing all Mems

- List < MEMS > nTsl : the sub-list of $L M$ containing untreated Mems

- List < Mems > slLMwAP : the sub-list of $L M$ containing Mems for which the absolute position is known

- List < Mems > slLMwORP : the sub-list of $L M$ containing Mems for which a relative position is known and the absolute position is not known

- List $<$ Mems $>N_{i}$ : the sub-list of nearby Mems of the $M e m s_{i}$

- List $<M E M S>N u A P_{i}$ : the sub-list of nearby Mems of the $M e m s_{i}$ having no known absolute posi- 
tion

- PositionR posR $\left(M_{i} / M_{j}\right)$ : The relative position of the $M e m s_{i}$ in the landmark associated with the $M e m s_{j}$

- Position A pos $A\left(M_{i}\right)$ the absolute position of $M e m s_{i}$

- Mems $_{i}$ : the current item (in process)

- $M e m s_{k}$ : the neighbor element being processed

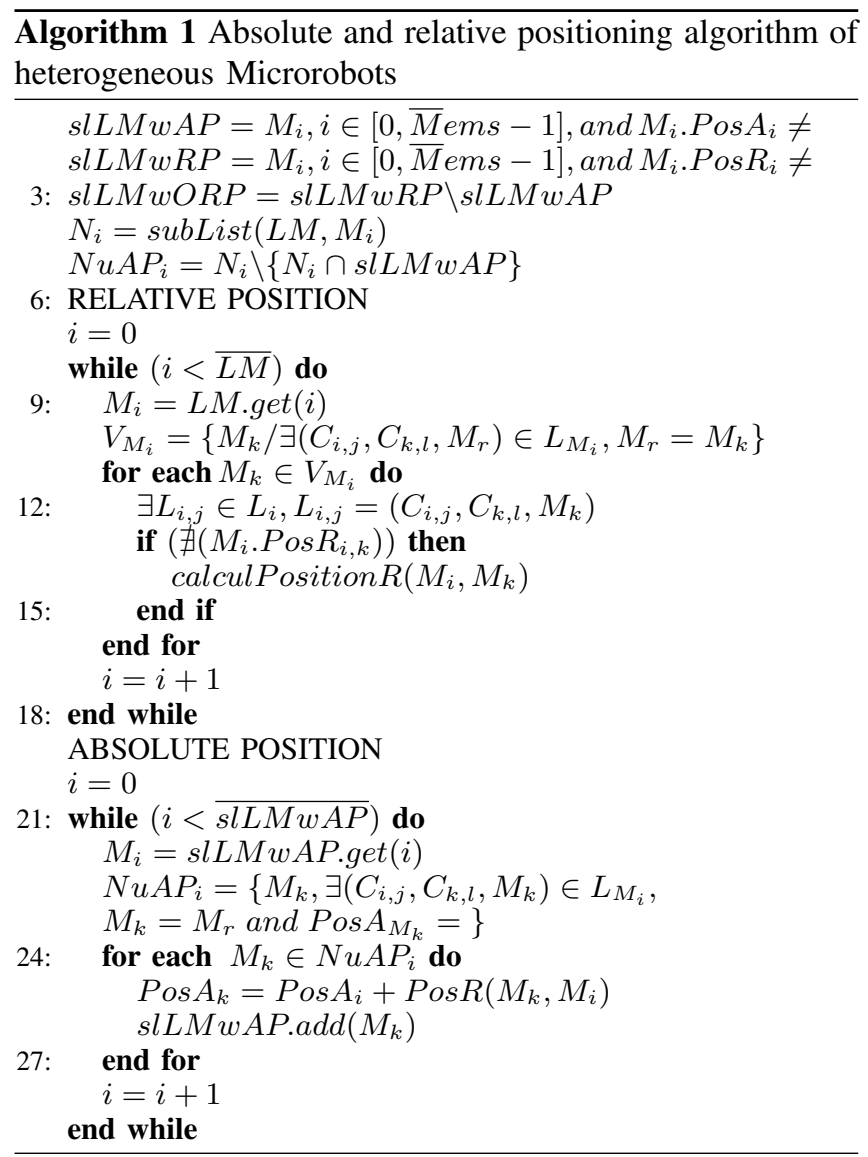

Figure .4 present an example of our Mems distributed system, on an orthogonal grid, with each Mems having a maximum of 4 neighbours. The initial configuration present our system at the begining, in each ralated component, we have a Mems with an absolute position. In the final configuration, each Mems now have and absolute position, wich have been calculated for those who did not previously have an absolute position.

\section{ReAlizATIONS AND TESTS}

In this section we present experimental results that illustrate the validation of our algorithm. We have developed Java simulations, which model 2D MEMS. Each Mems is modeled in 2D circular, With on its surface 4 connectors regularly spaced. This number of 4 connectors allows to define up to 4 physical neighbors for each Mems. When there is a connection between two nearby Mems, they can then exchange information. The communications between
Mems are made by contact. Our simulations tests are in 2D dimension. The sets are generated in a random way. Due to the circular geometric structure and the number of neighbors, we perform our tests on an orthogonal grid where each cell is a square of length equal to the diameter of a circular MEMS, and contains at most a MEMS. In case every Mems possesses 6 neighbors, we can also have a hexagonal grid in the form of honeycomb, where every Mems is registered in a regular hexagon. All tested MEMS are homogeneous and the determined positions are in cartesian coordinates. The positioning of elements is relative and/or absolute, depending on the initial state of the system.

The tests of number of configuration 1 - 3 of of Table III are functional validation tests.

They validate: The successful completion of the relative or absolute positioning algorithm of a homogeneous system of Mems communicating by contact. The good realization consists of the ending and the correctness of the calculated result. Accepted configurations correspond to all combinations of relations with the immediate neighborhood for the coherent types of links with the relative positions. The calculation of relative positions between elements in contact and connected (by link) The spread of the calculation of the absolute position within a connected component The validation of the consistency of the positions calculated with regard to links The validation of the ending and the calculation of its convergence The test of $n$ of configuration 4 represents a test of the coherence of the connections by reports to the relative positions. This test has the same parameters as the test of number of configuration 3; with the only difference of one relative position incoherence, of this fact, the number of global positions at the end the test is 18 and not 22 because of the inconsistency which limits the distribution of the global position to the other Mems. Table TABLE III and figures Fig. 5 present the result of passage on the scale of our algorithm. Unlike existing studies that position few micro-robots, our work goes beyond [3] which positions up to 10,000 micro-robots. We evaluate our algorithm on sets of Mems whose number varies from 10 to 700,000 for densities ranging from 0.1 to 0.95 .

\section{CONCLUSION}

In this work, we proposed a first approach of mixed positioning, absolute (global coordinate system) and relative ( local landmark), in systems of micro-robots with strong density. The systems of considered coordinates are mixed by manipulating the Cartesian coordinates and also those polar.

The model systems address the grid and honeycomb structures in 2D or 3D. Modeled Mems are heterogeneous or homogenous and have irregular or regular structures, like their connectors. The irregular structure concerns the geometry and specific functionalities of connectors of a Mems 

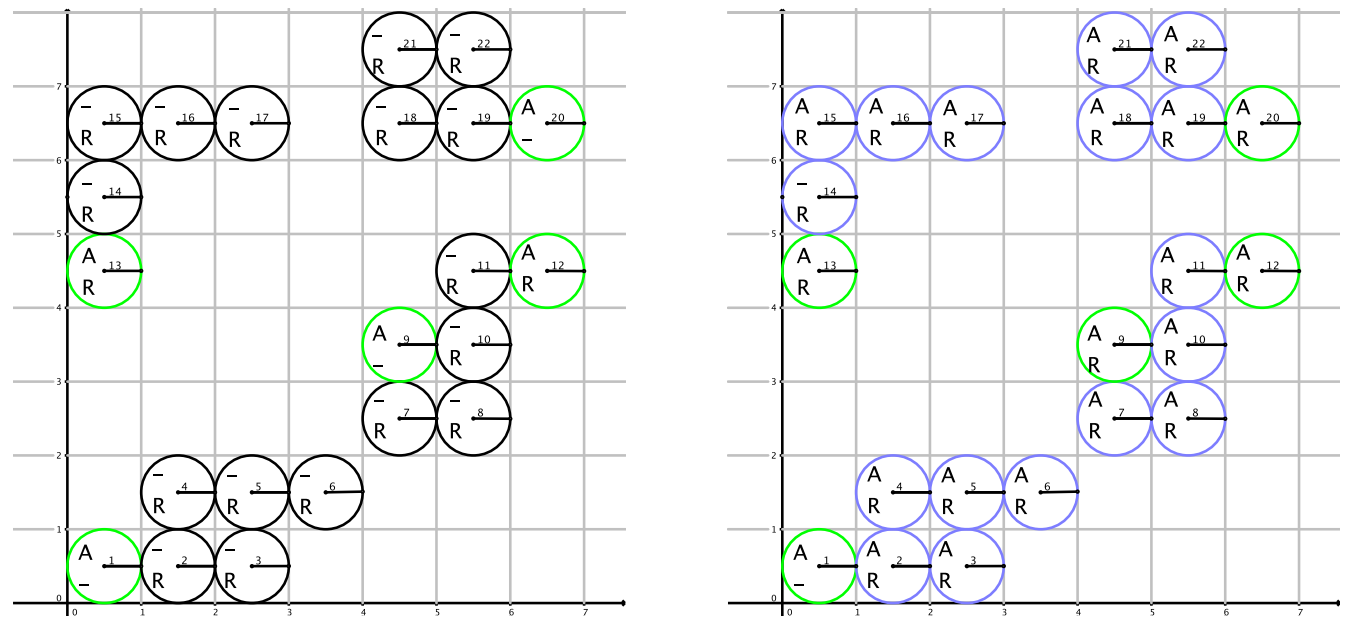

Figure 4. Initial an final configuration of $22 \mathrm{Mems}$ distributed on an orthogonal grid, in 4 connected components, each having initially at least one Mems with and absolute position. Applying our algorithm aims to calculate the absolute position of all Mems.

Table II

BOARD OF THE FUNCTIONAL VALIDATION TESTS: SHOWS THE BEHAVOUR OF OUR ALGORITHM IN DIFFERENTS SITUATIONS. WE VALIDATE THE CALCULATION OF ABSOLUTE AND RELATIVE POSITIONS, THE NORMAL BEHAVIOUR IN CASE OF INCOHENRENT LINKS, THE GOOD SPREAD IN A RELATIVE COMPONENT, AND MESURE OF CALCULATION TIME.

\begin{tabular}{|c|c|c|c|c|c|c|c|c|c|c|c|c|c|c|c|c|}
\hline \multirow{3}{*}{$\begin{array}{l}\text { Test } \\
\text { Id }\end{array}$} & \multicolumn{12}{|c|}{ Distribution } & \multicolumn{4}{|c|}{ Complexity } \\
\hline & \multicolumn{2}{|c|}{$\begin{array}{l}\text { Numbers } \\
\text { of } \\
\text { global } \\
\text { posi- } \\
\text { tions }\end{array}$} & \multicolumn{2}{|c|}{$\begin{array}{l}\text { Numbers } \\
\text { of } \\
\text { relative } \\
\text { posi- } \\
\text { tions }\end{array}$} & \multicolumn{2}{|c|}{$\begin{array}{l}\text { Numbers } \\
\text { of } \\
\text { global } \\
\text { and } \\
\text { relative } \\
\text { posi- } \\
\text { tions }\end{array}$} & \multicolumn{2}{|c|}{$\begin{array}{l}\text { Numbers } \\
\text { of po- } \\
\text { sitions } \\
\text { neither } \\
\text { global } \\
\text { nor } \\
\text { relative }\end{array}$} & \multicolumn{2}{|c|}{$\begin{array}{l}\text { Numbers } \\
\text { of } \\
\text { Mems } \\
\text { with } \\
\text { links } \\
\text { incon- } \\
\text { sistent }\end{array}$} & \multicolumn{2}{|c|}{$\begin{array}{l}\text { Numbers } \\
\text { of } \\
\text { related } \\
\text { compo- } \\
\text { nents }\end{array}$} & $\begin{array}{l}\text { processing } \\
\text { time }\end{array}$ & $\begin{array}{l}\text { maximum } \\
\text { number of } \\
\text { elements }\end{array}$ & $\begin{array}{l}\text { Actual } \\
\text { number } \\
\text { of } \\
\text { elements }\end{array}$ & $\begin{array}{l}\text { occupation } \\
\text { rate }\end{array}$ \\
\hline & initial & final & initial & final & initial & final & initial & final & initial & final & initial & fina & $\begin{array}{l}\text { calculation } \\
\text { time }\end{array}$ & $1 * c * n b m c$ & & $\begin{array}{l}\text { actual } \\
\mathrm{nb} / \mathrm{nb} \\
\text { cells }\end{array}$ \\
\hline 1 & 1 & 40 & 1 & 40 & 0 & 40 & 38 & 0 & 0 & 0 & 1 & 1 & $<1 \mathrm{~ms}$ & 64 & 40 & 0.625 \\
\hline 2 & 0 & 0 & 1 & 0 & 0 & 0 & 39 & 0 & 0 & 0 & 1 & 1 & $<1 \mathrm{~ms}$ & 100 & 40 & 0.4 \\
\hline 3 & 5 & 22 & 0 & 22 & 0 & 22 & 17 & 0 & 0 & 0 & 5 & 5 & $<1 \mathrm{~ms}$ & 64 & 22 & 0.34375 \\
\hline 4 & 5 & 18 & 0 & 18 & 0 & 18 & 17 & 0 & 1 & 1 & 5 & 5 & $<1 \mathrm{~ms}$ & 64 & 22 & 0.34375 \\
\hline
\end{tabular}

- positioning calculation time for a density of 0.1

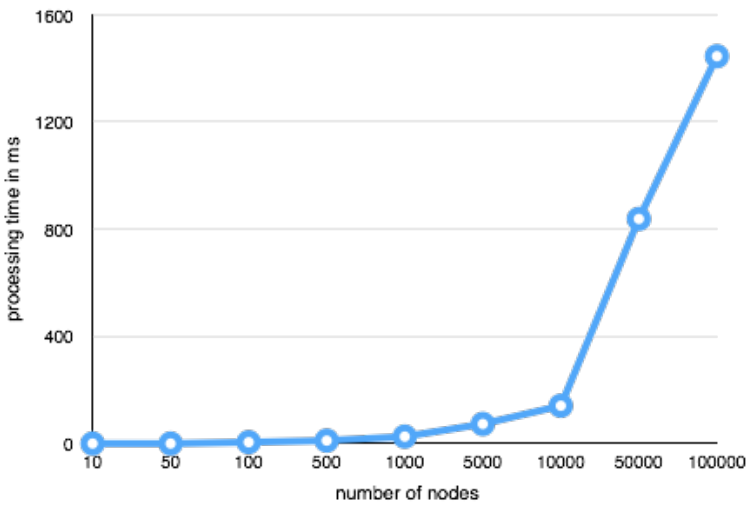

positioning calculation time for a density of 0.7

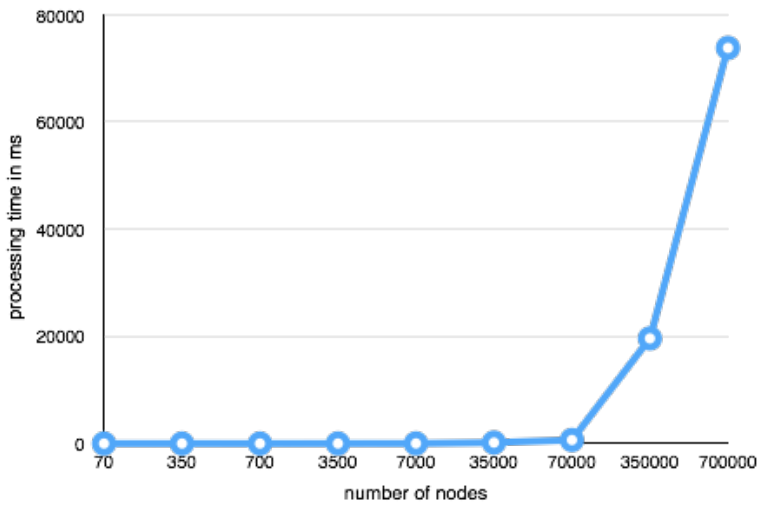

Figure 5. Curves of the calculation time of the relative and absolute positions in a MEMS system for densities of 0.1 and a number of MEMS ranging from 10 to 100,000 , and a density of 0.7 for a number of MEMS ranging from 70-700000 
Table III

TABLE SHOWING CALCULATION TIMES OF THE RELATIVE AND ABSOLUTE POSITIONS OF MEMS FOR DENSITIES 0.1 AND 0.7 FOR A NUMBER OF MEMS RANGING FROM 100 TO 1000000

\begin{tabular}{|c|c|c|c|c|c|c|c|c|c|}
\hline Number of MEMS for a density of 0.1 & 10 & 50 & 100 & 500 & 1000 & 5000 & 10000 & 50000 & 100000 \\
\hline Calculation time of the positions of all Mems (ms) & 0 & 0 & 5 & 11 & 26 & 73 & 141 & 838 & 1445 \\
\hline \hline Number of MEMS for a density of 0.7 & 70 & 350 & 700 & 3500 & 7000 & 35000 & 70000 & 350000 & 700000 \\
\hline Calculation time of the positions of all Mems (ms) & 0 & 5 & 3 & 14 & 23 & 180 & 660 & 19612 & 73802 \\
\hline
\end{tabular}

(or micro-robot) or specific and intelligent features of Mems (memorization, communication, movement, activations).

The algorithm of proposed positioning calculates the individual positionings of heterogeneous micro-robots communicating by contact. These micro-robots have knowledge of the physical contact with their immediate neighborhood and manipulate in a hierarchical way, and the reception of messages and those positions relative and absolved from the same immediate neighborhood.

The hierarchy considered by the neighborhood in contact, by the link made by received messages, the superiority of the knowledge of the global positioning on that of the local positioning, it leads to the proposal of a convergent algorithm with a linear complexity.

The realized tests validate functionally the heterogeneousnesses of modelling in the various scales of the system of micro-robots, via Mems and their associated connectors. The calculated positioning is absolute and relative and expresses himself in polar and Cartesian coordinates.

The reached scale reaches the million positioned intelligent micro-robots. According to the density testing between $10 \%$ and $70 \%$ of a space, the respective calculations of 100000 and 700000 micro-robots do not exceed $75 \mathrm{~s}$.

The tracks in this work are numerous. The obtained results, very encouraging, lead us to pursue this work and propose a distributed and collaborative computing positioning and integrating these libraries of positioning systems for micro-simulation systems such as VisibleSim.

Besides, all the irregular or regular geometrical configurations, and those functional were not estimated in this work. It's the same for the three-dimensional configurations of the system of Mems, to which we could add the temporal dimension necessary for the dynamic reconfigurations of system.

\section{ACKNOWLEDGMENT}

This project has been performed in cooperation with the Labex ACTION program (contract ANR-11-LABX0001-01). It has been also supported by the Ministère de l'Enseignement Suprieur et de la Recherche.

\section{REFERENCES}

[1] Nicolas Boillot, Dominique Dhoutaut, and Julien Bourgeois. Using nano-wireless communications in micro-robots applications. In Proceedings of ACM The First Annual International Conference on Nanoscale Computing and Communication, page 10. ACM, 2014.
[2] Julien Bourgeois and Seth Copen Goldstein. Distributed intelligent mems: Progresses and perspectives. Systems Journal, IEEE, 9(3):1057-1068, 2015.

[3] Stanislav Funiak, Padmanabhan Pillai, Jason Campbell, and Seth C Goldstein. Internal localization of modular robot ensembles. page 6, 2007.

[4] Seth C Goldstein and Todd C Mowry. Claytronics: A scalable basis for future robots. page 6, 2004.

[5] Robert Grabowski and Pradeep Khosla. Localization techniques for a team of small robots. In Intelligent Robots and Systems, 2001. Proceedings. 2001 IEEE/RSJ International Conference on, volume 2, pages 1067-1072. IEEE, 2001.

[6] Tran-Dang Hoa, Nicolas Krommenacker, and Patrick Charpentier. Localization algorithms based on Hop counting for wireless nano-sensor networks. In Fifth International Conference on Indoor Positioning and Indoor Navigation, IPIN2014, Busan, South Korea, October 2014.

[7] Josep Miquel Jornet and Ian F Akyildiz. The internet of multimedia nano-things. Nano Communication Networks, 3(4):242-251, 2012.

[8] Ling Mao, Jiapin Chen, Zhenbo Li, and Dawei Zhang. Relative localization method of multiple micro robots based on simple sensors. International Journal of Advanced Robotic Systems, 10, 2013.

[9] Mike Peasgood, Christopher Clark, and John McPhee. Localization of multiple robots with simple sensors. In Mechatronics and Automation, 2005 IEEE International Conference, volume 2, pages 671-676. IEEE, 2005.

[10] Renato Tinós, Luis E Navarro-Serment, and Christiaan JJ Paredis. Fault tolerant localization for teams of distributed robots. In Intelligent Robots and Systems, 2001. Proceedings. 2001 IEEE/RSJ International Conference on, volume 2, page 10611066. IEEE, 2001.

[11] Hoa Tran-Dang, Nicolas Krommenacker, and Patrick Charpentier. Localization algorithms based on hop counting for wireless nano-sensor networks. In Indoor Positioning and Indoor Navigation (IPIN), 2014 International Conference on, pages 300-306. IEEE, 2014.

[12] Mark Yim, Wei-Min Shen, Behnam Salemi, Daniela Rus, Mark Moll, Hod Lipson, Eric Klavins, and Gregory S Chirikjian. Modular self-reconfigurable robot systems [grand challenges of robotics]. Robotics \& Automation Magazine, IEEE, 14(1):43-52, 2007. 